\title{
Eficácia da absorção de impacto do Protetor Bucal na proteção de estruturas: Uma revisão sistemática dos Estudos de Elementos Finitos
}

\author{
Effectiveness of the impact absorption of Mouthguard in the protection of structures: A systematic \\ review of Finite Element Studies
}

Eficacia de la absorción de impactos del Protector Bucal en la protección de estructuras: Una revisión sistemática de los Estudios de Elementos Finitos

Recebido: 21/01/2022 | Revisado: 25/01/2022 | Aceito: 02/02/2022 | Publicado: 04/02/2022

\author{
Victor Paes Dias Goncalves \\ ORCID: https://orcid.org/0000-0001-6469-5735 \\ São Leopoldo Mandic, Brasil \\ E-mail: victor.dias.paes@gmail.com \\ Thayna Pereira Rangel \\ ORCID: https://orcid.org/0000-0003-0421-0163 \\ Universidade Estadual do Norte Fluminense Darcy Ribeiro, Brasil \\ e-mail: thaynarangel01@gmail.com \\ Eduardo Henrique Silva Wolf \\ ORCID: https://orcid.org/0000-0001-7894-9732 \\ Universidade Metodista de Piracicaba, Brasil \\ e-mail: wolfengcae@gmail.com \\ Fabiano Capato de Brito \\ ORCID: https://orcid.org/0000-0002-1786-9656 \\ São Leopoldo Mandic, Brasil \\ E-mail: fabianocbrito@gmail.com \\ Eduardo Cláudio Lopes de Chaves e Mello Dias \\ ORCID: https://orcid.org/0000-0002-4541-1095 \\ São Leopoldo Mandic, Brasil \\ E-mail: eduardodias@geati.com.br
}

\begin{abstract}
Resumo
As injurias orofaciais são extremamente comuns em esportes de contato e a cultura da competitividade fornece um ambiente onde as lesões são frequentes, principalmente os impactos em elementos dentários, conhecidos como traumatismo dentário. Esse trabalho tem por objetivo fazer uma revisão sistemática da literatura científica sobre a eficácia dos protetores bucais na redução das lesões orofaciais durante atividades esportivas. Após a triagem dos bancos de dados e remoção de duplicatas, 46 estudos foram identificados. Após a seleção do título e leitura cuidadosa dos resumos foram realizadas exclusões dos artigos que não seguiam critérios de inclusão e exclusão, e a revisão sistemática foi realizada com 13 artigos. Os achados da presente revisão sistemática com estudos realizados por MEF avaliando o comportamento biomecânico de tensões, distribuição de estresse e as deformações indicam que o uso do PB reduz os riscos de lesões orofaciais durante as atividades esportivas e que a espessura de $3 \mathrm{~mm}$ ou $4 \mathrm{~mm}$ parecem ser mais adequadas.
\end{abstract}

Palavras-chave: Traumatismo dentário; Protetor bucal; Revisão sistemática; Odontologia do esporte.

\begin{abstract}
Orofacial injuries are extremely common in contact sports and the culture of competitiveness provides an environment where injuries are frequent, especially impacts on dental elements, known as dental trauma. This work aims to make a systematic review of the scientific literature on the effectiveness of mouthguards in reducing orofacial injuries during sports activities. After screening the databases and removing duplicates, 46 studies were identified. After selecting the title and carefully reading the abstracts, articles that did not meet the inclusion and exclusion criteria were excluded, and the systematic review was performed with 13 articles. The findings of the present systematic review with studies carried out by FEM evaluating the biomechanical behavior of tensions, stress distribution and deformations indicate that the use of BP reduces the risk of orofacial injuries during sports activities and that a thickness of $3 \mathrm{~mm}$ or $4 \mathrm{~mm}$ seems to be most suitable.
\end{abstract}

Keywords: Dental trauma; Mouthguard; Systematic review; Sport dentistry. 


\begin{abstract}
Resumen
Las lesiones orofaciales son extremadamente comunes en los deportes de contacto y la cultura de la competitividad proporciona un ambiente donde las lesiones son frecuentes, especialmente los impactos en los elementos dentales, conocidos como trauma dental. Este trabajo tiene como objetivo realizar una revisión sistemática de la literatura científica sobre la efectividad de los protectores bucales en la reducción de lesiones orofaciales durante la práctica deportiva. Después de examinar las bases de datos y eliminar los duplicados, se identificaron 46 estudios. Después de seleccionar el título y leer atentamente los resúmenes, se excluyeron los artículos que no cumplían con los criterios de inclusión y exclusión, y se realizó la revisión sistemática con 13 artículos. Los hallazgos de la presente revisión sistemática con estudios realizados por FEM que evalúan el comportamiento biomecánico de tensiones, distribución de esfuerzos y deformaciones indican que el uso de BP reduce el riesgo de lesiones orofaciales durante la actividad deportiva y que un espesor de $3 \mathrm{~mm}$ o $4 \mathrm{~mm}$ parece ser más adecuado
\end{abstract}

Palabras clave: Trauma dental; Protector bucal; Revisión sistemática; Odontología deportiva.

\title{
1. Introdução
}

Atualmente, Lesões nas extremidades superiores são extremamente comuns em esportes de contato, como futebol, basquete, handebol e os demais esportes de artes marciais. A cultura da competitividade fornece um ambiente onde as lesões são frequentes.

O traumatismo dentário é uma das injúrias que ocorrem com frequência durante a prática de esportes, podendo ocasionar perdas irreparáveis que afetam diretamente o desempenho físico do atleta (Badel et al., 2010). Cohenca et al., (2007) relataram que a incidência é maior em elementos dentários na região anterior de maxila de adolescentes e que os atletas experimentam uma lesão dentária durante a participação em esportes, pelo menos uma vez na vida.

O método de elementos finitos (MEF) foi desenvolvida no início dos anos 60 para resolver problemas estruturais no campo aeroespacial. O MEF é agora uma ferramenta bem aceita e poderosa na pesquisa científica. Ele foi adaptado da área de engenharia e é amplamente aplicado na área de Odontologia, sendo de grande utilidade na previsão de absorção e dissipação de impacto em diversas especialidades (Patil V et al., 2020).

Desta forma, esse trabalho tem por objetivo fazer uma revisão sistemática da literatura científica sobre a eficácia dos protetores bucais na redução das lesões orofaciais durante atividades esportivas.

\section{Metodologia}

\section{Protocolo}

Esta revisão sistemática foi realizada de acordo com as diretrizes PRISMA (Preferred Reporting Items for Systematic Review and Meta-Analysis) (http://www.prisma-statement.org).

\section{Questão focada}

A presente revisão sistemática foi realizada a fim de para responder à seguinte questão focada: “'Os PB são eficazes na redução dos riscos de lesões orofaciais durante as atividades esportivas".

\section{Fontes de informação e pesquisa}

Uma revisão sistemática da literatura foi realizada para analisar a capacidade do protetor bucal em absorver e dissipar tensões gerada por impacto em métodos de elementos finitos, que foi definido como 1) tensão e 2) deformação. Foi realizada uma extensa busca de artigos, apenas com artigos publicados antes de novembro 2021, nas bases de dados PubMed, Scopus, Web of Science, Latino-americana e Literatura Caribenha em Ciências da Saúde (LILACS) (Tabela 1). O processo de seleção está descrito na Figura 1. Não foram aplicadas datas ou restrições de idioma. Os termos MeSH "Mouthguard" foram usados (Tabela 1). Sinônimos MeSH, termos relacionados e termos livres foram incluídos (Tabela 1). Os termos foram combinados para 
refinar os resultados da pesquisa. Os títulos e resumos dos artigos identificados foram avaliados de forma independente por dois investigadores para determinar se cumpriam os critérios de inclusão para a revisão. A busca eletrônica foi complementada por uma busca manual na lista de referências dos artigos utilizados.

\section{Critério de eleição}

Os contornos dos critérios de inclusão, de acordo com a população, intervenções, comparações e desfechos (PICOS), foram realizados da seguinte forma:

População (P): Estruturas da Face

Intervenção (I): Protetor bucal.

Comparação (C): Controle do protetor bucal ou sem protetor bucal.

Resultado (O): tensão e deformação

Desenho do Estudo (S): Ensaio clínico randomizado.

\section{Critério de inclusão}

Estudos com Método de elementos Finitos

\section{Critério de exclusão}

Estudo mecânicos, sem condição de controle, capítulo de livro, revisão sistemática ou meta-análise e sem dados completos para análise.

\section{Seleção de estudos e coleta de dados}

Todos os artigos identificados eletronicamente foram digitalizados por título e resumo. Os artigos que apareceram em mais de uma busca na base de dados foram considerados apenas uma vez. Dois examinadores (ECLCMD e VPDG) realizaram o processo de pesquisa de forma independente. Em caso de discrepância, a decisão era tomada por consenso com um terceiro autor (FCB). Textos completos foram obtidos para todos os artigos identificados e considerados potencialmente relevantes. 
Quadro 1: Base de dados e Estratégia de Pesquisa.

\begin{tabular}{|c|c|}
\hline Base de Dados & Estratégia de Pesquisa \\
\hline \multicolumn{2}{|r|}{ PubMed } \\
\hline $\begin{array}{l}\text { \#1 (finite } \\
\text { elements) }\end{array}$ & $\begin{array}{l}\text { \#2 (mouthguard) OR (Mouth Protector)) OR (Protector, Mouth)) OR (Protectors, Mouth)) OR (Mouth Pieces, } \\
\text { Protective)) OR (Mouth Piece, Protective)) OR (Piece, Protective Mouth)) OR (Pieces, Protective Mouth)) OR } \\
\text { (Protective Mouth Piece)) OR (Protective Mouth Pieces)) OR (Mouthpieces, Protective)) OR (Mouthpiece, Protective)) } \\
\text { OR (Protective Mouthpiece)) OR (Protective Mouthpieces)) OR (Mouth Guards)) OR (Guard, Mouth)) OR (Guards, } \\
\text { Mouth)) OR (Mouth Guard)) }\end{array}$ \\
\hline \multicolumn{2}{|r|}{ Scopus } \\
\hline $\begin{array}{l}\text { \#1 TITLE-ABS- } \\
\text { KEY (finite } \\
\text { elements) }\end{array}$ & 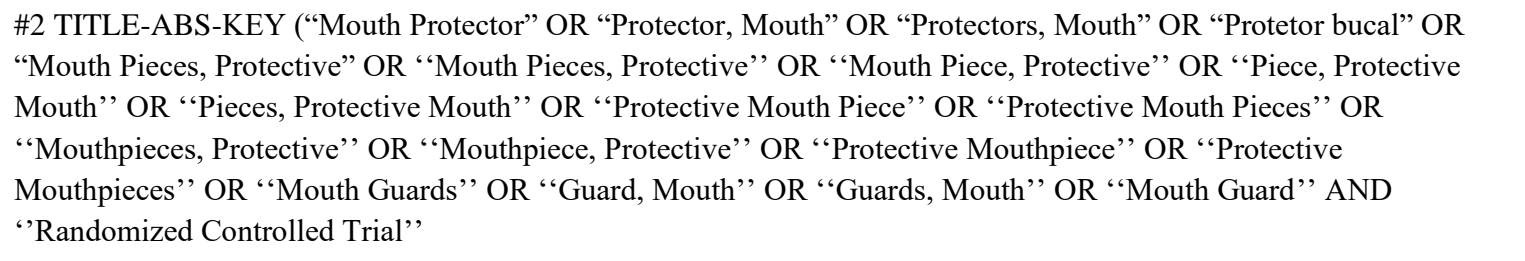 \\
\hline \multicolumn{2}{|r|}{ Web of Science } \\
\hline $\begin{array}{l}\# 1 \mathrm{TOPIC}= \\
\text { (finite elements) }\end{array}$ & 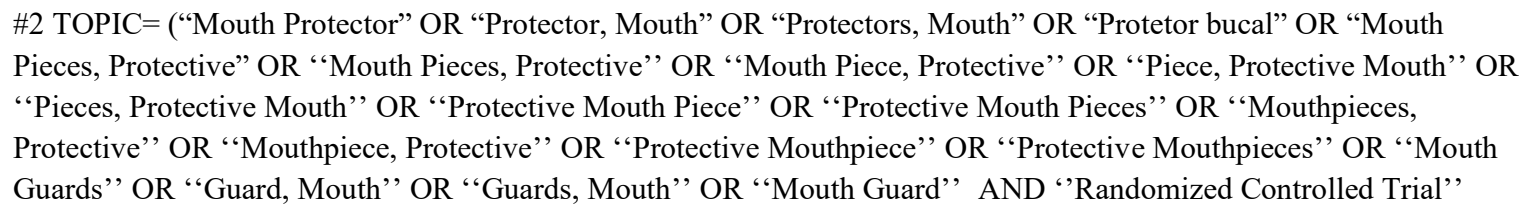 \\
\hline \multicolumn{2}{|r|}{ Latin American e Literatura Caribenha em Ciências da Saúde (LILACS) } \\
\hline $\begin{array}{l}\# 1 \text { (MH: finite } \\
\text { elements) }\end{array}$ & $\begin{array}{l}\text { \#2 (MH: (mouthguard) OR (Mouth Protector)) OR (Protector, Mouth)) OR (Protectors, Mouth)) OR (Mouth Pieces, } \\
\text { Protective)) OR (Mouth Piece, Protective)) OR (Piece, Protective Mouth)) OR (Pieces, Protective Mouth)) OR } \\
\text { (Protective Mouth Piece)) OR (Protective Mouth Pieces)) OR (Mouthpieces, Protective)) OR (Mouthpiece, Protective)) } \\
\text { OR (Protective Mouthpiece)) OR (Protective Mouthpieces)) OR (Mouth Guards)) OR (Guard, Mouth)) OR (Guards, } \\
\text { Mouth)) OR (Mouth Guard)) ) }\end{array}$ \\
\hline
\end{tabular}

Fonte: Autores.

\section{Resultados}

\section{Diagrama de fluxo dos critérios de pesquisa e seleção de literatura}

Após a triagem dos bancos de dados e remoção de duplicatas, 46 estudos foram identificados (Fig. 1). Após a seleção do título e leitura cuidadosa dos resumos, 30 estudos foram excluídos por não abordarem na sua metodologia o uso do MEF. Outros três estudos foram excluídos após a leitura dos textos completos: dois por avaliar diferença entre projetos do PB (Verissimo et al., 2015; Otani et al., 2018) e um por avaliar diferentes materiais de reforço (Tribst et al., 2021). Foram então incluídos 13 estudos nesta revisão. 
Figura 1: Fluxograma de Pesquisa.
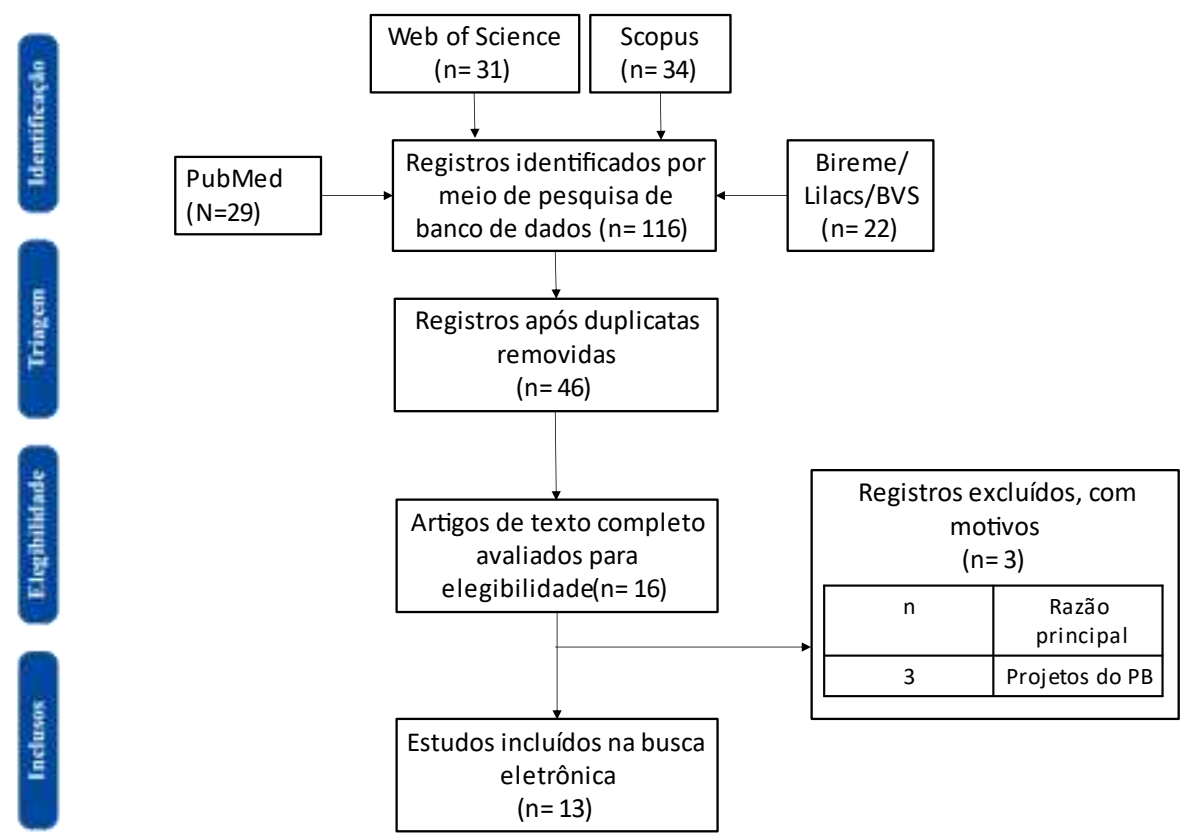

Fonte: Autores.

As características dos 13 estudos selecionados estão listadas na Tabela 2. Todos atenderam aos critérios de inclusão, entre os anos de 2015 e 2021. Dos 13 estudos, foram observados análises com diferentes estruturas: incisivo central (Poblete et al., 2012; Verissimo et al., 2015; Giailain et al., 2016; Verissimo et al., (2016); incisivo central com antagonista (Verissimo et al., 2016); incisivo com anquilose (Borges et al., 2020); incisivo central reabilitado com faceta dentária (Bragrança et al., 2021); incisivo com braquete ortodôntico (Alves et al., 2020); incisivo com canino impacto( Firmiano et al., 2019); implante dentário (Carvalho et al. 2018); articulação tempo-mandibular (Tribst et al., 2020) e crânio (Tribst et al., 2018; Tribst et al., 2018).

Quadro 2: Resultados.

\begin{tabular}{|c|c|c|c|c|}
\hline Autor & Estrutura & Protetor & Simulação & Conclusão \\
\hline $\begin{array}{l}\text { Poblete et al., } \\
(2012)\end{array}$ & Incisivo Central & $4 \mathrm{~mm}$ & $\begin{array}{l}\text { Cargas de } 500,1000 \text { e } \\
1500 N .\end{array}$ & $\begin{array}{l}\text { Ao se comparar o modelo controle com o } \\
\text { modelo provido de PB houve uma absorção } \\
\text { de tensões em até } 50 \% \text {; }\end{array}$ \\
\hline $\begin{array}{l}\text { Verissimo et } \\
\text { al., }(2015)\end{array}$ & Incisivo Central & $\begin{array}{c}2 \mathrm{~mm} ; 3 \mathrm{~mm} \\
4 \mathrm{~mm} ; 5 \mathrm{~mm} ; 6 \mathrm{~mm}\end{array}$ & $\begin{array}{l}\text { Impacto dinâmico não } \\
\text { linear realizado em } \\
\text { que um objeto rígido } \\
\text { atingiu o modelo a } 1 \mathrm{~m} \\
\text { / s }\end{array}$ & $\begin{array}{l}\text { PB promoveu menores tensões do que o } \\
\text { controle; não houve diferença significativa } \\
\text { nos picos de tensões e na absorção de choque } \\
\text { entre os PB que tinham } 4 \text { a } 6 \mathrm{~mm} \text { de } \\
\text { espessura. }\end{array}$ \\
\hline $\begin{array}{l}\text { Giailain et al., } \\
\text { (2016) }\end{array}$ & Incisivo Central & $\begin{array}{l}1 \mathrm{~mm} ; 2 \mathrm{~mm} \\
3 \mathrm{~mm} ; 4 \mathrm{~mm}\end{array}$ & $\begin{array}{l}\text { A esfera de borracha a } \\
0,5 \mathrm{~mm} \text { de distância da } \\
\text { estrutura a } \text { ser } \\
\text { impactada } \\
\text { diâmetro com } \\
\text { coroa, para } \quad \text { a } \\
\text { velocidade inicial: de } \\
5 \mathrm{~m} /\end{array}$ & $\begin{array}{l}\text { As tensões de compressão e tração } \\
\text { encontradas no esmalte diminuiu com o } \\
\text { aumento da espessura do PB. Entretanto, a } \\
\text { diferença entre o PB de } 3 \mathrm{~mm} \text { e o de } 4 \mathrm{~mm} \\
\text { foi mínimo. }\end{array}$ \\
\hline $\begin{array}{l}\text { Verissimo et } \\
\text { al., }(2016)\end{array}$ & Incisivo Central & $6 \mathrm{~mm}$ & $\begin{array}{l}\text { Pendulo impactado } \\
\text { em } 3 \text { ângulos: } 90^{\circ} \text { com } \\
\text { Steel ball }\end{array}$ & $\begin{array}{l}\text { O PB personalizado tinha uma capacidade } \\
\text { de absorção de choque atingindo } 95 \% \text { com } \\
\text { base em medições de deformação. }\end{array}$ \\
\hline $\begin{array}{l}\text { Verissimo et } \\
\text { al., (2016) }\end{array}$ & $\begin{array}{l}\text { Incisivo Central com } \\
\text { Antagonista }\end{array}$ & $3 \mathrm{~mm}$ & $\begin{array}{l}\text { Objeto de impacto } \\
\text { rígido (bola de aço) foi } \\
\text { simulado, } \quad \text { em }\end{array}$ & $\begin{array}{l}\text { O contato antagonista com o PB diminui o } \\
\text { deslocamento de uma força de impacto. Os } \\
\text { efeitos preventivos dos PB podem ser mais }\end{array}$ \\
\hline
\end{tabular}




\begin{tabular}{|c|c|c|c|c|}
\hline & & & $\begin{array}{l}\text { velocidade inicial a } 1 \\
\mathrm{~m} / \mathrm{s}(3,6 \mathrm{~km} / \mathrm{h})\end{array}$ & $\begin{array}{l}\text { benéficos se apoiados por antagonistas em } \\
\text { contato com os dentes. }\end{array}$ \\
\hline $\begin{array}{l}\text { Carvalho et al. } \\
\text { (2018) }\end{array}$ & $\begin{array}{l}\text { Implante Dentário } \\
\text { (Hexágono externo e } \\
\text { Cone Morse) }\end{array}$ & $4 \mathrm{~mm}$ & $\begin{array}{l}\text { Impacto, em que a } \\
\text { esfera metálica rígida } \\
\text { colidiu ao modelo em } \\
1 \mathrm{~m} / \mathrm{s}\end{array}$ & $\begin{array}{l}\text { O uso de um PB tem eficácia significativa na } \\
\text { absorção de choque em pacientes } \\
\text { reabilitados com implantes dentários sendo } \\
\text { capaz de absorver } 40 \% \text { a } 46 \% \text { da energia } \\
\text { causada pelo impacto no implante dentário, } \\
\text { sem diferença entre as conexões. }\end{array}$ \\
\hline $\begin{array}{l}\text { Tribst et al., } \\
(2018)\end{array}$ & $\begin{array}{l}\text { Crânio sem considerar } \\
\text { Oclusão }\end{array}$ & $1 \mathrm{~mm} ; 2 \mathrm{~mm} ; 4 \mathrm{~mm}$ & $\begin{array}{l}\text { Carga }(500 \mathrm{~N}) \text { foi } \\
\text { aplicada no dente } \\
\text { canino com uma bola }\end{array}$ & $\begin{array}{l}\text { Ao analisar conjunto de dentes, a região de } \\
\text { impacto é a mais comprimida em todos os } \\
\text { grupos e reflete zonas de tração em áreas } \\
\text { distintas da maxila e mandíbula, sugerindo } \\
\text { que os incisivos superiores poderiam sofrer } \\
\text { tanto dano como o canino direito. Esse } \\
\text { resultado ajuda a explicar que os incisivos } \\
\text { superiores são os dentes mais afetados, pois } \\
\text { mesmo sem impactá-los diretamente, } \\
\text { participam da dissipação do estresse. }\end{array}$ \\
\hline $\begin{array}{l}\text { Tribst et al., } \\
(2018)\end{array}$ & $\begin{array}{ll}\text { Crânio } & \text { considerando } \\
\text { Oclusão } & \end{array}$ & $4 \mathrm{~mm}$ & $\begin{array}{l}\text { Soco específico com } \\
\text { luva luta de boxe, e o } \\
\text { valor de } 1000 \mathrm{~N} \text { em } 3 \\
\text { eixos: Jab no incisivo } \\
\text { central superior; } \\
\text { punho cruzado no arco } \\
\text { zigomático; Uppercut } \\
\text { na sínfise Mandibular }\end{array}$ & $\begin{array}{l}\text { Os autores relataram que o uso de um PB } \\
\text { alivia as tensões de tração na face, } \\
\text { independentemente da local da lesão / } \\
\text { impacto. Os impactos diretos nas regiões } \\
\text { dentárias apresentaram os valores de } \\
\text { estresse mais agressivos e o uso de um PB } \\
\text { evidenciou a eficácia. }\end{array}$ \\
\hline $\begin{array}{l}\text { Firmiano et al. } \\
(2019)\end{array}$ & $\begin{array}{l}\text { Incisivo Central com } \\
\text { canino impactado }\end{array}$ & $3 \mathrm{~mm}$ & $\begin{array}{l}\text { Objeto de impacto } \\
\text { rígido (bola de aço) foi } \\
\text { simulado, em } \\
\text { velocidade inicial a } 1 \\
\mathrm{~m} / \mathrm{s}(3,6 \mathrm{~km} / \mathrm{h})\end{array}$ & $\begin{array}{l}\text { O PB poderia efetivamente reduzir } \\
\text { concentrações de tensão compressiva e de } \\
\text { tração nas estruturas cortical e osso } \\
\text { trabecular causado pela presença de um } \\
\text { canino impactado ou de uma cavidade } \\
\text { deixada pela cirurgia de extração. }\end{array}$ \\
\hline $\begin{array}{l}\text { Alves et al., } \\
(2020)\end{array}$ & $\begin{array}{l}\text { Incisivo Central com } \\
\text { Braquete r suporte } \\
\text { metálico e cerâmico }\end{array}$ & $3 \mathrm{~mm}$ & $\begin{array}{l}\text { Objetivo de aço, em } \\
\text { um raio de } 10 \mathrm{~mm} \text {, } \\
\text { numa velocidade } \\
\text { inicial de } 1 \mathrm{~m} / \mathrm{s} \text {, }\end{array}$ & $\begin{array}{l}\text { O tipo dos braquetes ortodônticos alteram as } \\
\text { distribuições de tensões e deformações nos } \\
\text { dentes durante o impacto. Os braquetes } \\
\text { cerâmicos geraram tensões maiores do que } \\
\text { os braquetes metálicos. Os protetores bucais } \\
\text { reduziram as tensões e deformações, } \\
\text { independentemente da composição do } \\
\text { braquete. }\end{array}$ \\
\hline $\begin{array}{l}\text { Borges et al., } \\
(2020)\end{array}$ & $\begin{array}{l}\text { Incisivo } \\
\text { Anquilosado }\end{array}$ & $4 \mathrm{~mm}$ & $\begin{array}{l}\text { Uma esfera de aço de } \\
35 \mathrm{~mm} \text { aplicada, numa } \\
\text { velocidade inicial de } 1 \\
\mathrm{~m} / \mathrm{s}\end{array}$ & $\begin{array}{l}\text { O uso de MG é benéfico para dentes } \\
\text { saudáveis e anquilosados, pois atua } \\
\text { amortecendo as tensões geradas no osso, } \\
\text { dentina, esmalte e ligamento periodontal. No } \\
\text { entanto, pacientes com dente anquilosado } \\
\text { são mais propensos a fraturas radiculares }\end{array}$ \\
\hline $\begin{array}{l}\text { Tribst et al., } \\
(2020)\end{array}$ & ATM & $4 \mathrm{~mm}$ & $\begin{array}{l}\text { 0-500N durante } 1 \mathrm{~m} / \mathrm{s} \text {, } \\
\text { aplicada por uma } \\
\text { esfera de aço de } 35 \\
\mathrm{~mm}\end{array}$ & $\begin{array}{l}\text { Os resultados mostram que a presença de PB } \\
\text { causa diminuição nas magnitudes de tensões } \\
\text { no côndilo e no disco articular para todos os } \\
\text { tipos de oclusão. A distribuição de tensões } \\
\text { foi semelhante entre os diferentes tipos de } \\
\text { oclusões. }\end{array}$ \\
\hline $\begin{array}{l}\text { Bragança et } \\
\text { al., (2021) }\end{array}$ & Incisivo Central & $4 \mathrm{~mm}$ & $\begin{array}{lr}\text { Cabeça } & \text { numa } \\
\text { Superfície } & \text { Rígida } \\
\text { numa } & \text { velocidade } \\
\text { inicial de } 1 \mathrm{~m} / \mathrm{s}\end{array}$ & $\begin{array}{l}\text { A presença de PB de } 4 \mathrm{~mm} \text { reduziu } \\
\text { substancialmente as tensões de impacto nos } \\
\text { laminados cerâmicos e nas estruturas } \\
\text { dentais. O contato do dente antagonista } \\
\text { promoveu melhor distribuição de estresse e } \\
\text { reduziu os níveis de estresse no dente } \\
\text { traumatizado. }\end{array}$ \\
\hline
\end{tabular}

Fonte: Autores. 


\section{Discussão}

Todos os estudos incluídos nesta revisão sistemática utilizaram o MEF. A característica essencial deste método é que podem ser produzidos dados operacionais confiáveis e em tempo reduzido, sendo de grande importância a multidisciplinaridade entre a Odontologia e a Bioengenharia.

Atualmente, o copolímero de etileno e acetado de vinila (EVA) é o material mais empregado para confecção de PB por apresentar boa capacidade de absorção do impacto e distribuição de tensões que são atenuadas de uma área menor para outra maior (Coto et al., 2009).

É ideal que para confecção de um PB, ele detenha características específicas, dentre elas: uma boa acomodação às estruturas bucais, ser confortável, de fácil higienização, permitir a respiração, a fala e a deglutição. Mas principalmente, seguir dois princípios: possuir espessura adequada para proteção dos impactos sofridos e não atrapalhar desempenho esportivo.

Em relação a espessura adequada para proteção dos impactos sofridos, apenas três pesquisas realizadas buscaram analisar a diferença das espessuras do PB (Verissimo et al., 2015; Giailain et al., 2016; Tribst., 2018). Os estudos apontam um consenso entre si: a recomendação da espessura PB de 3 a 4 mm. Verissimo et al. (2015) justificaram a escolha pela espessura pela relação espessura/conforto; Giailain et al. (2016) demonstram que o aumento acima de 3 mm não apresentou melhora significante, desta forma, elimina necessidade um PB com maior espessura. Por outro lado, Tribst et al. (2018) demonstraram que espessuras menores de $1 \mathrm{~mm}$ e $2 \mathrm{~mm}$ apresentaram resultados significantemente inferiores aos apresentados pelos PB de $4 \mathrm{~mm}$. Os demais autores avaliados nesta revisão sistemática definiram espessuras padrões em $3 \mathrm{~mm}$ e $4 \mathrm{~mm}$, exceto Verissimo et al. (2016) que utilizaram um PB com espessura de 6mm.

Dentre todas as estruturas avaliadas, o Incisivo Central foi a estrutura mais utilizada de forma isolada ou com modificações (Poblete et al., 2012; Verissimo et al., 2015; Giailain et al., 2016; Verissimo et al., 2016; Verissimo et al., 2016; Firmiano et al., 2019; Borges et al., 2020; Alves et al., 2020; Bragrança et al., 2021). Vilela et al. (2018) já destacavam que a intensidade das tensões nas raízes do dente impactado e adjacentes podem gerar sequelas irreversíveis, podendo ser necessário substituição por implantes dentários. Todos os treze artigos avaliados nesta revisão sistemática mostram redução significativa do impacto para todas as estruturas avaliadas.

Em relação a metodologia dos artigos, Verissimo et al. (2016); Carvalho et al. (2018) e Borges et al. (2020) realizaram a modelagem das estruturas através de modelagem direta no software ou por imagemJ (domínio público, National Institute Of Mental Health, Bethesda, Maryland). Diferente dos autores Poblete et al. (2012); Verissimo et al. (2015); Giailain et al. (2016); Verissimo et al. (2016); Tribst et al. (2018); Tribst et al. (2019); Firmiano et al. (2019); Alves et al. (2020); Tribst et al. (2020); Bragança et al. (2021) que realizaram a modelagem das estruturas de interesse baseadas em casos reais, de arquivos DICOM, aproximando a simulação da realidade.

A utilização dos PB não é unânime entre os atletas e sua incidência é baixa segundo pesquisas apresentadas por Andrade et al. (2010), Frontera et al. (2011) e Araujo et al. (2021). A principal causa é a crença que o PB irá prejudicar o desempenho esportivo. Vucic et al. (2016) destacaram que a queixa de desconforto dos atletas, principalmente na respiração, está relacionada a utilização de PB do tipo II (Ferve e Morde) e PB tipo I (Pré Fabricado), os quais não são bem recomendados. Desta forma, como apontado por Gonçalves et al., (2021) é indispensável a participação de cirurgiões-dentistas dentro de centros esportivos compondo a Equipe de Saúde, a fim de que estes profissionais orientem e esclareçam atletas, comissão técnica, clubes e federações desportivas quanto aos benefícios da relação esporte-Odontologia e da utilização de um protetor bucal individualizado confeccionado por Cirurgião - Dentista. 


\section{Considerações Finais}

Os achados da presente revisão sistemática com estudos realizados por MEF avaliando o comportamento biomecânico de tensões, distribuição de estresse e as deformações indicam que o uso do PB reduz os riscos de lesões orofaciais durante as atividades esportivas e que a espessura de $3 \mathrm{~mm}$ ou $4 \mathrm{~mm}$ parecem ser mais adequadas.

\section{Referências}

Andrade, R. A., Evans, P. L., Almeida, A. L., da Silva, J., Guedes, A. M., Guedes, F. R., Ranalli, D. N., Modesto, A., \& Tinoco, E. M. (2010). Prevalence of dental trauma in Pan American games athletes. Dental traumatology, 26(3), 248-253.

Araújo, F. M. de, Rabello, T. B., Berard, L. T., Coto, N. P., \& Dias, K. R. H. C. (2021). Prevalence of orofacial injuries and the level of knowledge about oral protection in a brazilian judo team. Research, Society and Development, 10(6), e57810616133.

Badel, T., Jerolimov, V., Pandurie, J. (2007). Dental/orofacial trauma in contact sports and intraoral mouthguard programmes. Kinesiol, 39(1):97-105

Borges, A., Dal Piva, A., Concílio, L., Paes-Junior, T., \& Tribst, J. (2020). Mouthguard Use Effect on the Biomechanical Response of an Ankylosed Maxillary Central Incisor during a Traumatic Impact: A 3-Dimensional Finite Element Analysis. Life (Basel, Switzerland), $10(11), 294$.

Bragança, G. F., Vilela, A., Soares, P., Tantbirojn, D., Versluis, A., \& Soares, C. J. (2021). Influence of ceramic veneer thickness and antagonist on impact stresses during dental trauma with and without a mouthguard assessed with finite element analysis. Dental traumatology, 37(2), 215-222,

Brown, J. C., Gardner-Lubbe, S., Lambert, M. I., van Mechelen, W., \& Verhagen, E. (2018). Coach-directed education is associated with injury-prevention behaviour in players: an ecological cross-sectional study. British Journal Of Sports Medicine, 52(15), 989-993.

Carvalho, V. F., Soares, P. B., Verissimo, C., Pessoa, R. S., Versluis, A., \& Soares, C. J. (2018). Mouthguard Biomechanics for Protecting Dental Implants from Impact: Experimental and Finite Element Impact Analysis. The International Journal Of Oral \& Maxillofacial Implants, 33(2), 335-343.

Cohenca, N., Roges, R. A., \& Roges, R. (2007). The incidence and severity of dental trauma in intercollegiate athletes. Journal of the American Dental Association (1939), 138(8), 1121-1126.

Coto, N. P., Meira, J. B., Brito e Dias, R., Driemeier, L., de Oliveira Roveri, G., \& Noritomi, P. Y. (2012). Assessment of nose protector for sport activities: finite element analysis. Dental traumatology,28(2), 108-113.

Firmiano, T. C., Oliveira, M., de Souza, J. B., Soares, C. J., Versluis, A., \& Veríssimo, C. (2019). Influence of impacted canines on the stress distribution during dental trauma with and without a mouthguard. Dental traumatology, 35(4-5), 276-284. 7

Frontera, R. R., Zanin, L., Ambrosano, G. M., \& Flório, F. M. (2011). Orofacial trauma in Brazilian basketball players and level of information concerning trauma and mouthguards. Dental traumatology,27(3), 208-216.

Gialain, I. O., Coto, N. P., Driemeier, L., Noritomi, P. Y., \& Dias, R. B. (2016). A three-dimensional finite element analysis of the sports mouthguard. Dental traumatology, 32(5), 409-415.

Gonçalves, V. P. D., Ferreira, T. T., Valentim, J. do R., Santos, T. B. dos, e Azevedo, M. M. A. (2021). Aspectos da odontologia do esporte em clubes de futebol no Rio de Janeiro. Lecturas: Educación Física y deportes, 26(277), 114-124

Otani, T., Kobayashi, M., Nozaki, K., Gonda, T., Maeda, Y., \& Tanaka, M. (2018). Influence of mouthguards and their palatal design on the stress-state of toothperiodontal ligament-bone complex under static loading. Dental traumatology, 34(3), 208-213.

Patil, V., Naik, N., Gadicherla, S., Smriti, K., Raju, A., \& Rathee, U. (2020). Biomechanical Behavior of Bioactive Material in Dental Implant: A ThreeDimensional Finite Element Analysis. The Scientific World Journal, $2020,2363298$.

Poblete, F. E. O., Noritomi, P. Y., Coto, N. P., Almeida, A. S.; Naclério-Homem, M. G. (2012). Análise por meio do método dos elementos finitos de um protetor bucal para atividades esportivas. RPG Rev. Pos-Grad, 19(4):159-164.

Tribst, J., Dal Piva, A., Borges, A., \& Bottino, M. A. (2019). Simulation of mouthguard use in preventing dental injuries caused by different impacts in sports activities. Sport Sci Health.; 15, 85-90.

Tribst, J., Dal Piva, A., Ausiello, P., De Benedictis, A., Bottino, M. A., \& Borges, A. (2021). Biomechanical Analysis of a Custom-Made Mouthguard Reinforced With Different Elastic Modulus Laminates During a Simulated Maxillofacial Trauma. Craniomaxillofacial Trauma \& Reconstruction, 14(3), 254-260.

Tribst, J., Dal Piva, A., Bottino, M. A., Kleverlaan, C. J., \& Koolstra, J. H. (2020). Mouthguard use and TMJ injury prevention with different occlusions: A three-dimensional finite element analysis. Dental traumatology, 36(6), 662-669.

Tribst, J., de Oliveira Dal Piva, A. M., Borges, A., \& Bottino, M. A. (2018). Influence of custom-made and stock mouthguard thickness on biomechanical response to a simulated impact. Dental traumatology, 34(6), 429-437.

Veríssimo, C., Bicalho, A. A., Soares, P. B., Tantbirojn, D., Versluis, A., \& Soares, C. J. (2017). The effect of antagonist tooth contact on the biomechanical response of custom-fitted mouthguards. Dental traumatology, 33(1), 57-63.

Verissimo, C., Costa, P. V., Santos-Filho, P. C., Fernandes-Neto, A. J., Tantbirojn, D., Versluis, A., \& Soares, C. J. (2016). Evaluation of a dentoalveolar model for testing mouthguards: stress and strain analyses. Dental traumatology, 32(1), 4-13. 
Research, Society and Development, v. 11, n. 2, e51611226005, 2022

(CC BY 4.0) | ISSN 2525-3409 | DOI: http://dx.doi.org/10.33448/rsd-v11i2.26005

Verissimo, C., Costa, P. V., Santos-Filho, P. C., Tantbirojn, D., Versluis, A., \& Soares, C. J. (2016). Custom-Fitted EVA Mouthguards: what is the ideal thickness? a dynamic finite element impact study. Dental traumatology, 32(2), 95-102.

Verissimo, C., Santos-Filho, P. C., Tantbirojn, D., Versluis, A., \& Soares, C. J. (2015). Modifying the biomechanical response of mouthguards with hard inserts: A finite element study. American Journal Of Dentistry, 28(2), 116-120.

Vilela, A., Soares, P., Almeida, G. A., Veríssimo, C., Rodrigues, M. P., Versluis, A., \& Soares, C. J. (2019). Three-dimensional finite element stress analysis of teeth adjacent to a traumatized incisor. Dental traumatology, 35(2), 128-134. https://doi.org/10.1111/edt.12453. 